\title{
Investigation of delamination mechanisms in polymer coatings by scanning acoustic microscopy
}

\author{
I. Alig ${ }^{1^{*}}$, M. Bargmann ${ }^{1}$, H. Oehler ${ }^{1}$, D. Lellinger ${ }^{1}$, M. Wanner ${ }^{2}$, D. Koch ${ }^{2}$ \\ ${ }^{1}$ Deutsches Kunststoff-Institut, \\ Schlossgartenstr. 6, \\ 64289 Darmstadt, Germany \\ ${ }^{2}$ Fraunhofer Institut für Produktionstechnik und Automatisierung, \\ Nobelstrasse 12, \\ 70569 Stuttgart, Germany
}

\begin{abstract}
Scanning acoustic microscopy (SAM) was used to investigate disbonding, delamination and blister formation in polymer coatings for different layer structures and initial defect depths during exposure to a corrosive environment. The time evolution of disbonding and blister initiation and their growth was investigated by analysing the SAM images exposure to an electrolyte solution (Harrison solution).

From investigations of a model system of coatings on steel substrates, it was possible to differentiate between water and/or ion transport (i) through the coating and (ii) along the coating-steel interface. For samples without clear coat randomly distributed blisters appeared at the coating-steel interface after exposure to the electrolyte solution, irrespective of the location of initial defects. The random distribution of growing blisters is related to diffusion of water and/or ions through the coating and "nucleation" at weak points of the substrate or within the polymer. For samples with clear coat a propagating migration front along the coating-steel interface of 3-4 $\mu \mathrm{m}$ height - starting from initial defects - was detected. The linear propagation of this front cannot be explained by Fickian diffusion. Therefore, it is discussed in terms of an accelerated diffusion or crack growth kinetics. Since blistering starts only at sites, where the migration front has passed, the presence of a thin water layer at the coating-steel interface, the loss of adhesion and the following corrosive processes are prerequisites for the nucleation of blisters. The blister growth shows a square-root time dependence which was related to a diffusion controlled transport mechanism.
\end{abstract}

\section{Keywords:}

Scanning acoustic microscopy, coatings, delamination, disbonding, blisters, diffusion

\section{Short title:}

Investigation of delamination in coatings by SAM

\footnotetext{
* Corresponding author: ialig@dki.tu-darmstadt.de
} 


\section{Introduction}

The effective protection of steel substrates from corrosion is an important aspect in coating technology. Besides passivation of the substrate surface (inhibitive effect) and sacrificial protection (galvanic effect), barrier protection is one of the basic protective mechanisms of anticorrosive organic coatings [1]. The protective properties of barrier coating systems are known to depend on numerous factors, including the material properties of the polymer binders, pigmentation, layer structure and thickness, type of corrosive environment, type and pre-treatment of the substrate, the presence of initial defects or impacts etc. [1]. Although the general mechanisms preceding coating failure are basically understood, the exact cause and nature of coating breakdown is still in dispute [1]. The processes involved in coating failure are transport of water, ions and oxygen followed by adhesion loss and corrosive chemical reactions. The transport processes include water diffusion through the polymer matrix, along the coating-metal interface or through cracks [2 - 4] as well as migration of ions $[1-3,5]$. These mechanisms are assumed to be the ratedetermining steps for the decrease in adhesion strength between coating and substrate [1,6] ("weak wet adhesion"), cathodic disbonding, blistering, delamination [1 - 3, 5, 7 - 11] and rust creep [12].

Adhesion is of vital importance for anticorrosive coatings, since weak adhesion permits a loss in the coating's protective properties and corrosion of the metallic substrate. These processes finally result in coating failure $[1,6]$. Adhesion and deadhesion are interfacial phenomena occurring, in the specific case of coatings, at the coating-metal interface. Usually, for organic coatings adhesion is considered to consist of two factors (see [1] and references therein): (i) mechanical adhesion due to penetration of the coating into surface pits (anchoring) and (ii) chemical adhesion, where bonds are formed between metal and coating. Possible bonds are covalent and ionic (primary bonds), dipole and van-der-Waals (secondary bonds) and hydrogen bonds. It is generally believed that in coating-metal systems hydrogen or secondary bonds between the metal oxide layer and the polymer (e.g. polar groups) are dominant $[1,3]$. Adhesion can, for example, be improved by using coupling agents. Whereas, on the other hand, swelling and thermal cycling, the resulting mechanical stress, coating cracks and process-related water-soluble contaminants on the substrate negatively influence adhesion [13].

As stated above, the transport rate of water, oxygen and ions is assumed to control disbonding, delamination and blister formation. In general, one can differentiate between transport (i) through the coating and (ii) along the coating-metal interface. Therefore, we used a model system with different layer structures to differentiate between those possibilities (see chapter 2.2). The transport of dissolved oxygen and water is generally considered to be sufficient to sustain corrosive reactions and therefore not to be the rate determining process (see [1-3] and references). For example, the cathodic delamination of epoxy coatings was found to be most probably controlled by the interfacial transport of hydrated cations in a thin aqueous layer at the coating-steel interface [2]. Another mechanism discussed in literature is "weak wet adhesion" [1, 14], which refers to adhesion loss due to water molecules at the coating-metal interface replacing hydrogen bonds due to their hydrogen bonding ability, thereby reducing the attractive forces between coating and metal oxides [15]. Supposedly, the resulting thin water film between polymer and metal is regarded as a prerequisite for electrolytes entering the interface and initiating corrosive reactions. 
The mechanism of weak wet adhesion is a physical-chemical mechanism that is not limited to corrosive environments.

SAM does not provide direct information on the chemical reactions of cathodic disbonding and blistering, as it is only sensitive to differences in the investigated acoustic properties. For a detailed description of wet corrosion mechanisms we refer to reference [1]. While cathodic disbonding starts near defects, cathodic blistering can also be found on coatings without any evident defects. It is assumed that water penetrating the coating accumulates at sites of reduced adhesion or network density at the polymer-metal interface. An anode is initiated and a cathode develops in the vicinity. At the cathode dissolved oxygen is reduced, resulting in hydroxyl ions and other aggressive reaction products that attack the metal. Water-soluble contaminants on the metal as well as the water-soluble reaction products from the cathodic reaction lead to osmotic pressure between the interfacial region and the outside environment. Due to this the disbonded area fills with water and grows. Although corrosion and coating failure resulting from cathodic disbonding and blistering are issues of large practical relevance, the number of systematic studies on disbonding, delamination and damage kinetics of coatings on metal substrates [2, 3, $7-9,16]$ is limited. In these works experiments were carried out using a variety of coating materials and exposure to different types of corrosive media. Also, most research is concerned with epoxy-coating systems. For example, Crossen et al. [7] found for an epoxypolyamide coating that the widening of the delamination zone follows parabolic kinetics, which indicates a diffusion controlled process. Sorenson et al. [8] reported on the influence of substrate topography on cathodic disbonding of anticorrosive coatings. They found a dependence of the cation transport rate on the tortuosity of the substrate and measured a square-root dependence of the delaminated area on time.

As mentioned above, the delamination behaviour of many anticorrosive coatings has been found to be characterised by a square-root dependence between the extent of delamination and exposure time. In that case, the transport of cations is considered to be the rate determining process, therefore, a proportionality between mean diffusion length, $\bar{x}$, and diffusion time, $t$, has been assumed [3]:

$$
\bar{x} \propto \sqrt{D_{a p p} t}
$$

$D_{a p p}$ is an apparent diffusion coefficient, taking into account that the transport of the ions is facilitated by an electrical field gradient [2, 3] between anode and cathode resulting from the cathodic reaction [17]. This behaviour can be expressed using the Nernst-Planck equation. However, a novel approach uses [2] Fick's second law to estimate the apparent diffusion coefficient of ions along an ultrathin aqueous layer at the coating-metal interface. In the corresponding experiments an 'induction time' has been found, which is suggested to be the result of the transport processes of reactants through the coating.

In contrast, for polyurethane coatings we recently reported [9] finding an exponential growth (exponent between 1.3 and 1.5) of a blistering zone during a salt spray test. The exponential growth was related to a nucleation and growth mechanism. In Reference [16], exponents for the time-dependent growth of delaminations of up to 2.6, depending on substrate conditions, were reported. 
Using Fick's second law to estimate transport parameters for water and/or ions along an interface or the time dependence of delamination extent it has to be presumed that the diffusion coefficient of the species does not change over time [2]. Regarding the complex situation at a coating-metal interface this seems to be rather restrictive. For about 60 years it has been established by experiments and simulations $[18,19]$ that the diffusion properties of a penetrated polymer system can change with time as diffusion proceeds. Two mechanisms have been suggested [18]: (i) slow structural changes of the polymer and (ii) different, differential swelling of parts of the polymer sheet as penetrant enters. For 'history dependent' diffusion, it is assumed that the polymer chains reconfigure in order to accommodate the penetrating molecules. A rapid change of the equilibrium diffusion coefficient around the penetrant can lead to an actual 'diffusion front' with a sharp boundary. At this front, the highly swollen side is characterised by a high diffusion coefficient, whereas the non-swollen side exhibits a small diffusion coefficient. This behaviour is typical for glassy polymers or networks where the diffusion coefficient drops several orders of magnitude due to a solventinduced glass-to-rubber transition. This effect not only occurs for organic solvents, but is also evident in water absorption induced "hydroplastification" of polymers. Therefore, mass transport of water or solvents in glassy polymers has to be described by a diffusion equation taking into account moving boundaries. This type of diffusion is "anomalous" with respect to the classical Fickian diffusion [20]. The socalled "Case II" [20] or "Type II" transport [21, 22] is characterised by linear penetrant uptake with time and a constant velocity of the moving front, thus differing from the square-root time dependence of the penetrant uptake and the continuous concentration profiles in Fickian diffusion. To solve the differential equation for a "history-dependent" diffusion numerical methods have to be used. An alternative mechanism was discussed by Crank [19], relating the effect to strain-dependant diffusion, which also can lead to an "anomalous" penetrant diffusion. In this case, the sharp boundary between outer, swollen part and inner, non-swollen part is assumed to exert internal stresses, influencing the diffusion. Interestingly, there exists a close analogy between the structural changes involved at the swelling front and those occurring at a crazing front during mechanical deformation [23]. As a result of this analogy, similar boundary conditions and differential equations are used to describe swelling and crazing kinetics under mechanical stress [24].

This brief review of possible transport mechanisms and related experimental findings shows the lack of experimental information concerning diffusion along the coatingmetal interface. Time resolved experiments with sufficient spatial resolution are needed, to estimate transport parameters and to improve understanding regarding the kinetics of migration, disbonding/delamination and defect initiation and growth. The various methods having been used to examine deteriorating coating performance comprise classical methods like the 'cross cut adhesion test' or visual methods (e.g. removal of delaminated coating, measuring the rusted area underneath [3, 8]). More advanced methods, based on electrical potential, electrical impedance or acoustic properties of the coating are Scanning Kelvin Probe (SKP) [25 - 27], Electrochemical Impedance Spectroscopy (EIS) [6, 27, 28] and Scanning Acoustic Microscopy (SAM) $[25,26,29]$, respectively. However, a combination of different methods can provide complementary information [13, 30, 31].

SAM is a non-destructive method, which allows gathering depth-specific and time resolved information with a lateral resolution of some micrometers. Despite its 
advantages the application of SAM in coating research is still rare [9, 13, $29-34]$ : For example, Briggs and Addison et al. [32, 35] applied SAM for in-situ monitoring of cathodic disbonding and Crossen et al. [7] examined changes at the interface during cathodic disbonding of an epoxy-polyamide coating exposed to a $\mathrm{NaCl}$ solution. More recently, we applied SAM to investigate solvent- and water-borne $2 \mathrm{~K}$ polyurethane systems and UV-cured acrylic systems after simulated weathering.

In this study, SAM was used to investigate disbonding, delamination and blister formation in polymer coatings of different layer structures and different initial defects during exposure to a corrosive environment. The time evolution of migration of water and/or ions, disbonding and blister initiation and growth was studied by analysing the SAM images after exposure to electrolyte solution (Harrison solution). To this effect, new methods for the analysis of SAM images have been developed. Although SAM does not provide a direct measure of penetrant concentration, changes in the materials' acoustic properties allowed to extract information on surface profiles, propagating migration fronts and blistering and the underlying transport mechanisms. The results showed the usefulness of SAM for non-destructive investigation of timedependent processes in polymer coatings.

\section{Experimental}

\subsection{Scanning Acoustic Microscopy}

SAM works by focusing a short high frequency acoustic pulse - emitted by an ultrasonic transducer and focused by an acoustic lens - on a small area on a target object. The incident ultrasonic wave is scattered, absorbed, reflected or transmitted. Usually, the area of interest is located in the focal plane. For each position the reflected and/or the transmitted acoustic signals can be detected. The sample is scanned by moving the transducer until the entire region of interest has been investigated. Based on the actual operating mode a certain value from the received signal is assigned to the location investigated. Characteristic properties of the signal for each position (like amplitude and phase of the reflected signal in a defined time interval) are assembled into an image of the object. The contrast seen in the image is based on sound absorption, scattering and phase effects of the object and its geometry.

At interfaces between materials of different acoustic impedances an ultrasonic pulse is partly reflected and partly transmitted. The acoustic impedance $Z$ for a given material is defined as the product of sound velocity $c_{s}$ and density $\rho$ :

$$
Z=c_{s} \rho
$$

The fraction of amplitude reflected at the interface of two materials is proportional to the acoustic reflection coefficient $R$, which is given for perpendicular incidence by

$$
R=\frac{Z_{2}-Z_{1}}{Z_{1}+Z_{2}}
$$


$Z_{1}$ and $Z_{2}$ are the acoustic impedances of the two materials. Strong reflections occur if the impedances are sufficiently different. Therefore interfaces between water, air, steel and polymer can be clearly distinguished whereas interfaces between two polymer layers in most cases do not provide sufficient difference in acoustic impedance. In these experiments the good acoustic contrast between polymer coating and steel substrate results in a strong reflection from the substrate surface. From the time delay of the reflected echoes combined with the known sound velocity of the material, the thickness of the sample (profiling), the spacing of different layers (multi-layer systems of acoustically sufficiently different materials) and the positions of defects can be calculated.

Due to the viscoelastic properties of polymers, ultrasonic waves are attenuated considerably and the penetration depth is limited. However, since industrial polymer coatings utilised e.g. in the automotive industry are very thin, ultrasonic microscopy in the range of ca. $20-250 \mathrm{MHz}$ offers sufficient penetration depth and spatial resolution to examine such coatings for defects. Detectable defects comprise air and water filled blisters, delamination of the coatings, cracks and pigments [9].

For our investigations a SAM Type "Evolution II" by PVA TePla Analytical Systems $\mathrm{GmbH}$ was used in reflection mode. In the experiments presented ultrasonic transducers with a nominal frequency of $30 \mathrm{MHz}$ and $150 \mathrm{MHz}$ were used to examine the multi-layer coatings of a total thickness of approx. $15-75 \mu \mathrm{m}$, depending on the sample's layer composition. The maximum spatial resolution at $150 \mathrm{MHz}$ is in the range of $10 \mu \mathrm{m}$. During measurements the transducer tip and the samples were immersed in distilled water at room temperature. Further numerical data analysis was performed for selected signals.

\subsection{Model system}

The model layer composition consisted of rolled steel substrates of approximately $30 \times 20 \mathrm{~mm}^{2}$ coated with different combinations of primer, base coat and clear coat layers. For the coatings we used an commercial black base coat (Heliotherm-HydroBasislack Type 16-123-9731) by Bollig \& Kemper GmbH \& Co. KG of about $15 \mu \mathrm{m}$ thickness, an industrial clear coat (ProGloss Type FF99-0778) by BASF Coatings AG of about $40 \mu \mathrm{m}$ and a corrosion inhibiting primer applied by electrophoretic deposition (EPD) of $15 \mu \mathrm{m}$. The base coat is water-based with polyurethane and acrylic dispersion particles and a melamine component, the clear coat acrylate based. Since the investigated coatings are commercial systems, further details are not available.

The base and clear coat were applied separately by spray-coating and pre-cured at $80{ }^{\circ} \mathrm{C}$ for $10 \mathrm{~min}$ before the next layer was applied. The completed samples were then cured in an oven at $140^{\circ} \mathrm{C}$ for another 20 minutes. Into each sample a hole of $0.5 \mathrm{~mm}$ diameter with varying depth (see below) was drilled into the coating layer(s) to serve as well defined initial defect; the depth of the defects was checked with a profilometer. The defect depth was varied to get information on the transport processes in the different layers and along the interfaces. Furthermore, two different types of defects, a 'hole' and a 'scratch' (applied by a sclerometer) were compared. The sample edges were left free of coating or other sealants to allow penetration of the water and electrolytes from the edges. In addition, all samples provided sufficient surface area to study coating regions without any initial defect. 
The samples are named according to their layer structure from bottom to top (primer: P, base coat: BC and clear coat: CC). The depth of the defect D ('hole') is indicated by the number of affected layers from top to bottom (Fig. 1).

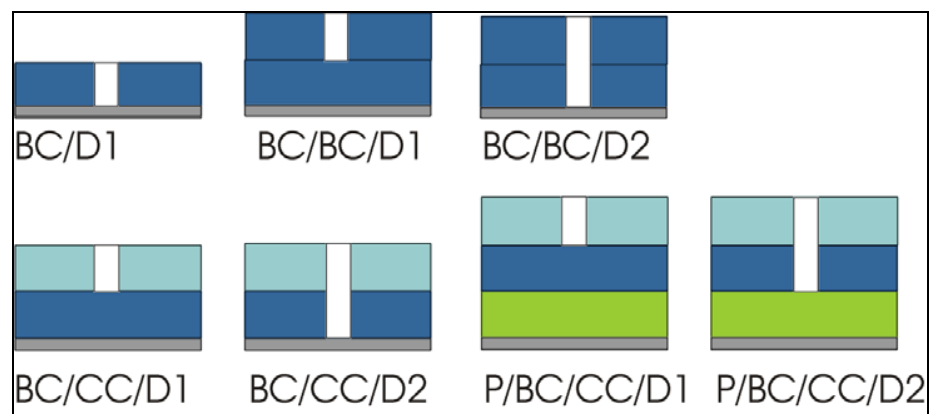

Fig.1: Layer structure of the model coatings. An explanation of the nomenclature can be found in the text.

To provide the corrosive environment the samples were immersed in Harrison solution $\left(5 \mathrm{~g} / \mathrm{l} \mathrm{NaCl}\right.$ and $35 \mathrm{~g} / \mathrm{l}\left(\mathrm{NH}_{4}\right)_{2} \mathrm{SO}_{4}$ in distilled water). Measurements were carried out after exposure intervals of approximately 24-62 hours. Accumulated immersion times of up to 500 hours provided sufficient time resolution to assess the delamination and damage mechanisms of interest. All experiments were carried out at room temperature.

\section{Results and Discussion}

\subsection{Dependence of damage mechanisms on coating layer structure}

The SAM images in Fig. 2 a - c and Fig. 3 a - d show typical examples of damage effects in polymer coatings after similar times of immersion (between about 250 to 280 hours) in Harrison solution. All samples in Fig. 2 are without clear coat, whereas all samples in Fig. 3 feature a layer of clear coat (CC) on top. Only the samples in Fig. $3 c$ and $3 d$ contain a primer $(P)$. Depending on the layer structure two different basic mechanisms of defect initiation can clearly be distinguished. 

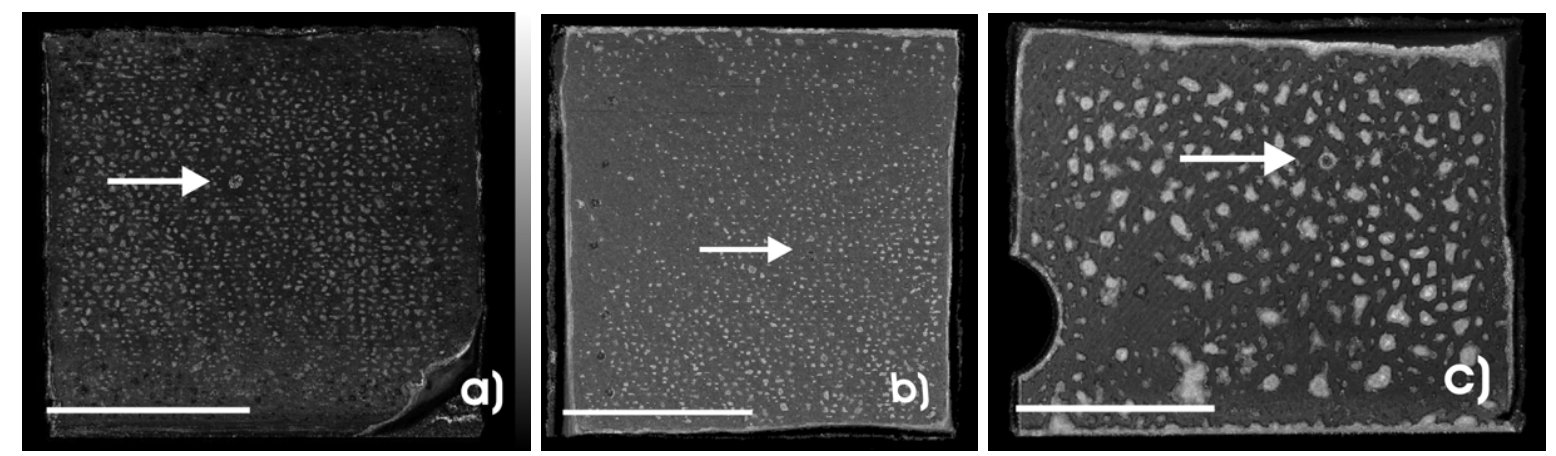

Fig. 2 a - c:

Ultrasonic images of samples without clear coat. a) BC/D1 after $267 \mathrm{~h} 54 \mathrm{~min}, \mathrm{~b}) \mathrm{BC/BC/D1}$ after $285 \mathrm{~h} 42 \mathrm{~min}$ and c) BC/BC/D2 after 246 h 12 min immersion in Harrison solution. White scale bars: $10 \mathrm{~mm}$. The arrows mark the initial defects (holes of $0.5 \mathrm{~mm}$ diameter).

For the samples without clear coat, shown in the SAM images in Fig. $2 \mathrm{a}-\mathrm{c}$, microblisters appeared uniformly over the sample area, irrespective of the location and depth of the initial defect. An analysis of the reflected ultrasonic signal at the blister sites reveals the presence of a strong substrate echo and a change in the time of flight of the echo in the range of $12-34$ ns compared to regions without blisters. Assuming the blisters to be water-filled this value corresponds to a height of 9 $25.5 \mu \mathrm{m}$. The height differences measured in a topographical evaluation (see Fig.4a) are also about $10-25 \mu \mathrm{m}$. These features indicate that the blisters are filled mainly with water, electrolytes and water-soluble corrosion products. Since the blisters disappear almost completely after the sample's removal from the immersion, only marginal amounts of solid corrosion products like rust are contained in the blisters. As will be shown below, the micro-blisters growth follows a power law dependence on time.

The 'spinodal' type of blister morphology and the growth kinetics has been linked to the idea of "nucleation and growth" for blister formation [9]. Nucleation and growth describes the kinetics of a phase separation (e.g. crystallisation or second order phase transition), where a nucleus for the new phase forms or "nucleates". Such a nucleus can either be formed spontaneously by fluctuations of the relevant order parameter or at a preferential site like boundaries or impurities. The nucleated phase grows until growth is inhibited or the structures merge. A detailed description can be found elsewhere [36, 37,38]. The similarity of blister formation - which in the case of very thin coatings is rather a $2 \mathrm{D}$ than a $3 \mathrm{D}$ process - to nucleation and growth is that a liquid phase (electrolyte/water) appears at the interface between polymer matrix and substrate and that this phase grows at the expense of its surrounding material. The origin of 'blister nucleation' can be both homogeneous due to fluctuations of the electrolyte concentrations or heterogeneous due to defects at the substrate or inhomogeneities in properties such as network density or adhesion strength. However, it has been stated that before the blistering can begin, water or electrolyte solution has to have penetrated the base coat and reached the substrate, so that a local loss of adhesion and disbonding can start [11]. Blister formation is assumed to take place in "weak spots" [25], that are presumably present due to manufacturing, but the exact reason why blisters form where they form is still under debate [1].

Considering that the first signs of blistering could be detected after about two days of immersion and that the blisters were statistically distributed, without any indication of a dependence on the location of the initial defect (white arrows in Fig. 2 and 3), a fast and uniform diffusion of the electrolyte solution through the polymer matrix of the 
base coat to the substrate region has to be assumed. This shows that the barrier properties of the base coat alone are not sufficient to protect the underlying substrate from exposure to water and corrosive solution or prevent damage mechanisms even if the coating were intact.
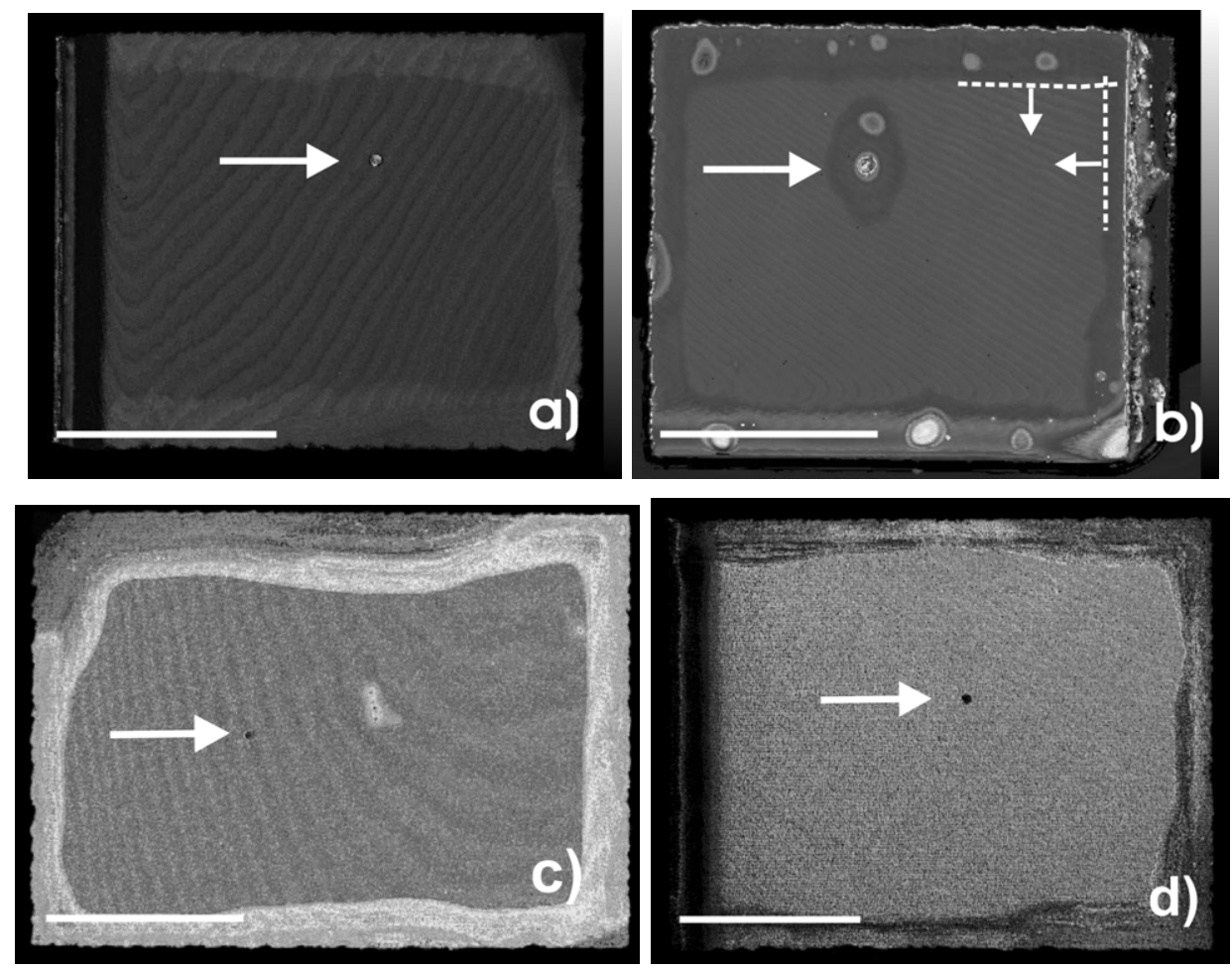

Fig. 3 a-d: Ultrasonic images of samples: a) BC/CC/D1 after $287 \mathrm{~h} \mathrm{12min}$, b) $B C / C C / D 2$ after 282 h $31 \mathrm{~min}$, c) $P / B C / C C / D 1$ after 285 h $42 \mathrm{~min}$ and d) $P / B C / C C / D 2$ after 273 h 07 min immersion in Harrison solution. White scale bars: $10 \mathrm{~mm}$. The arrows mark the initial defects.

The samples with clear coat (Fig. 3) showed quite different behaviour. No microblisters could be detected in a time interval of about $300 \mathrm{~h}$. On the other hand there was evidence of a sub-surface migration starting at the unprotected edges and the defect. Most probably water penetrates the interface between polymer and substrate (see Fig. 4a below). Other authors [1, 2, 3] found that water and oxygen penetrate coatings quickly, facilitating the beginning loss of adhesion by the reduction of the $\mathrm{O}_{2}$ to $\mathrm{OH}^{-}$lons, thereby creating the potential gradient that supports the diffusion of ions into the coating. As has been pointed out before, SAM is not able to differentiate between water and electrolyte solution. Therefore, in our experiments no conclusion regarding the exact nature of the penetrating liquid is possible. The SAM images in Fig. 3 clearly show a dependence of the observed effect on defect depth. The migration around the defect only occurred if the hole reached the substrate (Fig. $3 \mathrm{~b}$ ). Since none of the ultrasonic images of the other samples (the holes did not reach the metal substrate) showed any changes around the hole after up to 400 hours of Harrison immersion while all of them showed an effect along the sealant-free edges, it can be deduced that the effect occurs in the region of the substrate/coating interface, not at intermediate interfaces. This corresponds to similar results found in other research $[5,11]$.

By visual inspection at the sites where migration was detected by SAM no macroscopic delaminations (coating peeling from the substrate) was evident. Fig. $4 a$ 
shows an exemplary topographic profile of sample BC/CC/D2 after 388 hours of exposure supporting this. The $z$-profile $(\Delta h)$ of the sample was measured horizontally along the $y$-axis passing close to the initial defect. The position of the profile is indicated by the black line in SAM image in Fig. 4b.
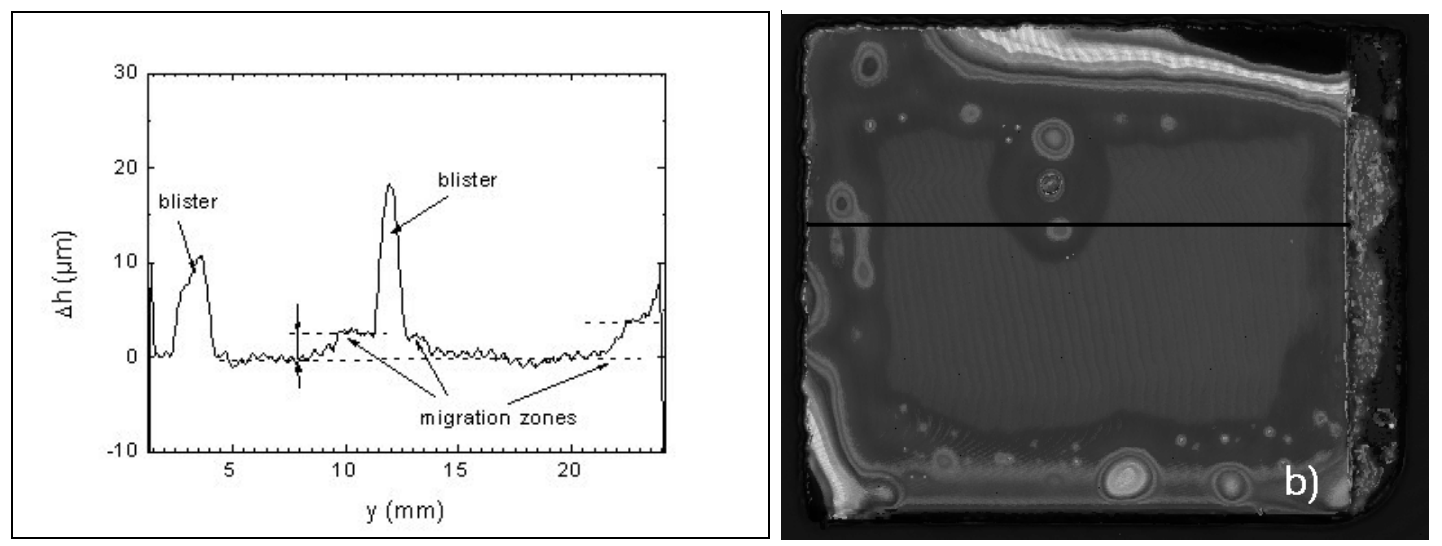

Fig. 4a-b: a) Topographic profile (cross section) of sample $B C / C C / D 2$ after immersion in Harrison solution for approximately $388 \mathrm{~h}$. The height difference $\Delta h$ is plotted along a line in y-direction. Different damage types can be seen: water/electrolyte migration zones on the right edge and near the defect (middle), and blisters on the left side and in the middle close to the initial defect. Figure $4 b$ shows the line along which the topography was evaluated.

The topographical evaluation was done using the time-of-flight information of the reflected ultrasonic signal from the sample surface (see Fig. 5). Since the coupling medium is water, the distance from the transducer to the sample surface can easily be calculated for every point $(x, y)$ on the sample surface using $s(x, y)=t(x, y) \cdot c / 2$, where $c$ is the ultrasonic velocity of water, and $t$ is the time-of-flight of the reflected signal. To correct for sample tilt and bending of the substrate, the distance information was used to calculate a "mean sample distance function" by fitting a quadratic function in $x$ and $y$ :

$s_{\text {mean }}(x, y)=a_{00}+a_{10} x+a_{01} y+a_{11} x y+\ldots+a_{22} x^{2} y^{2}$,

where $a_{00} . . a_{22}$ are coefficients. Only the non-delaminated inner area of the sample was used for this calculation. The height difference was then calculated by subtracting the mean sample distance function from the actual, measured distance:

$\Delta h(x, y)=s_{\text {mean }}(x, y)-s(x, y)$. 


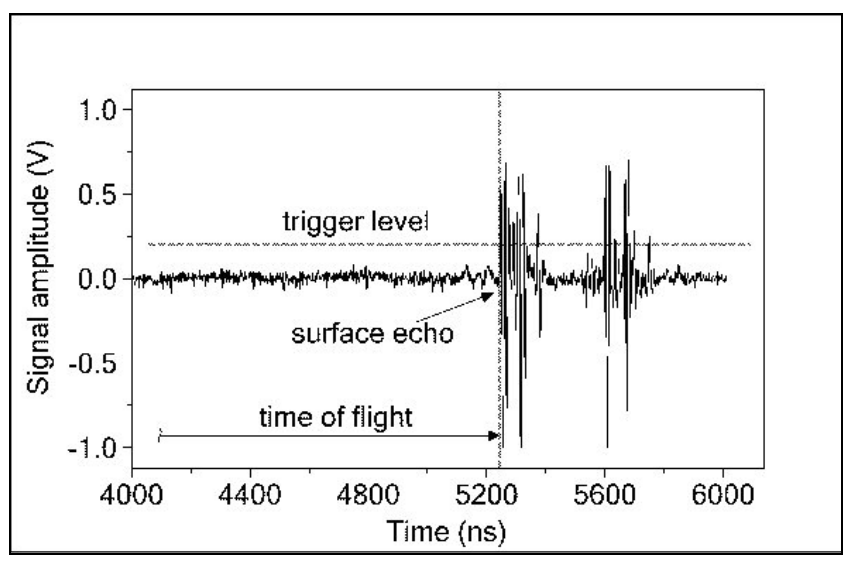

Fig. 5: $\quad$ Typical signal (A-scan) from the ultrasonic microscope. The time-offlight was measured from the start of the signal to the point when a certain trigger level was reached. The time-of-flight information was then used to calculate the height profile.

From left to right the cross section in Fig. 4a indicates a blister (left side), a blister close to the position of the initial defect (middle) which is surrounded by a migration zone and another migration region on the right side of the sample. At the right and left edge of the sample there is an indication of a slight elevation of the surface with a height of about 7-10 $\mu \mathrm{m}$ in the ultrasonic echo profile. This delamination is supported by visual inspection. In the migration zones the increase of the surface is about 3 - $4 \mu \mathrm{m}$ (see dotted lines in Fig. 4a). These zones can be interpreted as areas where an initial loss of adhesion through weak wet adhesion and possibly disbonding of the polymer coating from the substrate due to migration of water and dissolved $\mathrm{O}_{2}$ into the interface region took place [1]. In contrast the two blisters (left and middle) have a height of $10-25 \mu \mathrm{m}$.

From these results it can be concluded that the clear coat effectively shields underlying layers from exposure to electrolyte solution, so that no diffusion of solution into the coating through the coating bulk starting from the surface takes place. Hence, no micro-blisters can form. The barrier property of the clear coat is therefore extremely important for preventing damage under exposure. After an induction time during which only the migration could be detected nucleation and growth of blisters started in the migration regions for samples where no primer was used on the substrate. Samples featuring a layer of primer (P/BC/CC/D1 and P/BC/CC/D2) did not show any blisters at any time before the complete delamination of the coatings from the substrate, which can be attributed to an enhanced adhesion of the primer. In most experiments by other researchers samples without primer were used $[5,7,9$, $11,39]$, impeding a direct comparison of results.

The influence of the layer structure, specifically the absence of clear coat or primer, on the damage mechanisms holds true irrespective of the actual form of the defect. This was confirmed by experiments investigating samples featuring different types of defects (thin and broad scribes, hole or no initial defect at all). The geometry of the defect affects only the shape of the migration zone. A hole results in a roughly circular region expanding outward, while for scribes the front runs parallel to the scribe.

\subsection{Migration kinetics}


The migration kinetics were examined using a time series of SAM images taken after different times of exposure to Harrison solution. Fig. 6 a - $f$ shows such a time series for sample BC/CC/D2. The progression of fluid migration into the polymer due to continuous immersion can be seen. As has been deduced from Fig. 4 this migration of water results most likely in weak wet adhesion [1] and possibly an initial disbonding, but does not yet represent a delamination. The migration fronts in $x$ - and $y$-direction are indicated by white dotted lines and two short arrows denoted by $x$ and $y$ in the upper right corner of Fig. 6b. Some time after the water/electrolyte migration, delamination occurs. This delamination is represented by the black regions in the top right-hand corner and bottom left-hand corner of Figs. $6 \mathrm{c}-\mathrm{f}$. From the ultrasonic images an anisotropy in the propagation velocity of the migration fronts is apparent. 

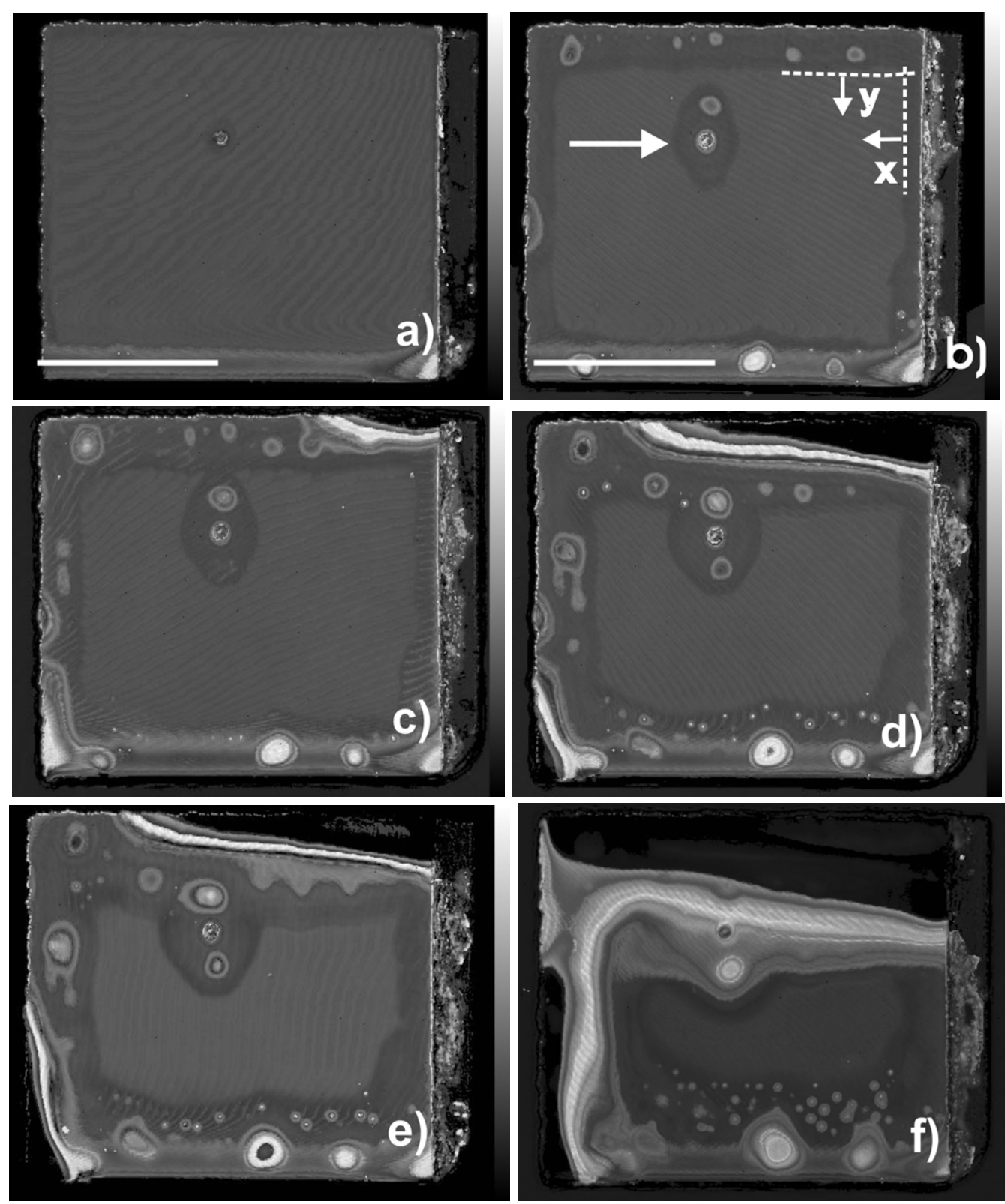

Fig. 6 a - f: $\quad$ Time series for sample BC/CC/D2. The ultrasonic images were taken after approx.: a) $201 h$, b) $282 h$, c) $325 h$, d) $403 h$, e) $439 h$ and f) $540 \mathrm{~h}$ of immersion in Harrison solution.

The $x$ - and $y$-positions of the migration fronts in the SAM images taken after different exposure times were measured vertically and horizontally for the upper and righthand edge of the sample and for the migration zone around the hole. The results can be seen in Fig. 7. Within the bounds of error, the data show a distinct grouping according to direction. The extent of the migration regions was found to depend linearly on time with different expansion velocities in $x$ - and $y$-direction. The data were fitted using a linear fit (here for the $x$-direction):

$$
x=v_{\text {migr }}\left(t-t_{0, \text { migr }}\right) \quad,
$$

where $v_{\text {migr }}$ is the migration velocity and $t_{0, m i g r}$ is the induction time that passes before any migration and possible disbonding can be observed. The resulting values for $v_{\text {migr }}$ are $1.8 \mathrm{~nm} / \mathrm{s}$ for the $x$-direction (horizontal) and $3.5 \mathrm{~nm} / \mathrm{s}$ for the $y$-direction (vertical). The induction times $t_{0, \text { migr }}$ for $x$ - and $y$-direction are 60 and 63 hours for the migration 
around the defect, and 80 and 90 hours for the edges. The differences between the induction times at the hole and along the edges are almost similar, which indicates the same induction process for both locations.

Induction times have also been found in other experiments, for example a diffusioncontrolled delamination process in a study on cathodic disbonding [8] and in a study of the influence of various parameters on cathodic delamination of a coating featuring a defect due to exposure to sea water, where the induction times were in the range of 84 - 180 hours [3]. In the same experiment the advancing delamination front's velocity was measured to be $2.32 \mathrm{~nm} / \mathrm{s}$ [3], which corresponds very well to the values measured in our experiments. In reference [2] the induction time is suggested to be controlled by the transport of reactants through the coating. However, no final explanation for the origin of induction time can be given here.

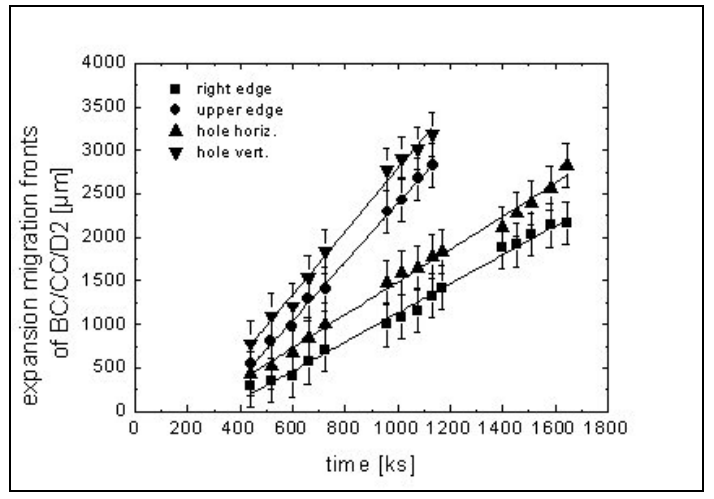

Fig. 7: $\quad$ Measured expansion of the migration fronts for sample BC/CC/D2. The measurements were taken in $x$-direction on the right edge of the sample and around the hole (horizontally), and in $y$-direction on the upper edge and around the hole (vertically).

The different migration velocities for $x$ - and $y$-direction on our sample lead to the conclusion that the properties of the substrate are relevant for the propagation of the migration front. This conclusion is supported by other works, where a significant effect of the substrate characteristics on disbonding and corrosion propagation has been reported $[8,16]$. In our case the different velocities observed are most likely a result of the rolling of the steel substrate that is part of the manufacturing process.
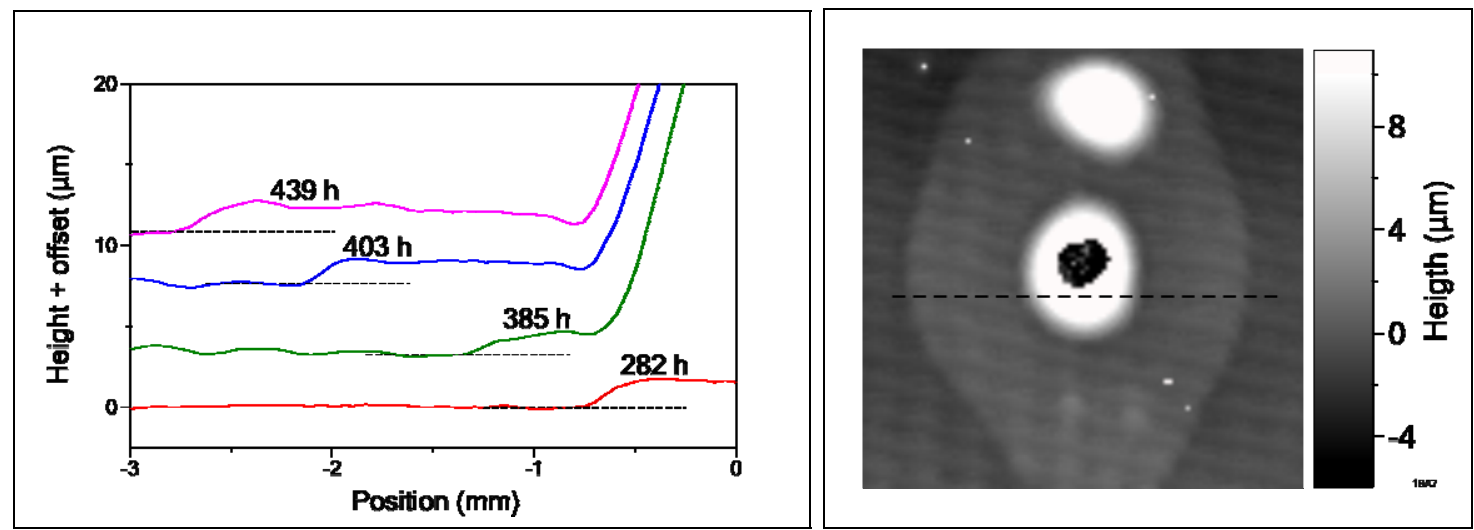

Fig.8a-b: a) Topographical profile of the migration front at different exposure times and b) the line for which the topographical profiles are displayed. The stepwise change by about $2 \mu \mathrm{m}$ is related to the propagating 
migration front, whereas the strong increase of the height on the right side is related to the blister growth.

The constant migration velocities and the stepwise change of the topographical profiles at the migration front in Fig. 8a suggest that the observed migration cannot be described by a classical Fickian diffusion mechanism. Although it is not possible to differentiate between water and electrolyte diffusion by SAM, the stepwise increase by about $2 \mu \mathrm{m}$ can be explained by a migration of a penetrant along the coatingmetal interface which most probably leads to swelling of the polymer near the metal surface. SAM investigation cannot exclude mechanisms like adhesion loss by 'weak wet adhesion' and cathodic disbonding. Parallel investigations with complementary methods could be helpful in future research. Combined experiments using SAM and SKP to investigate a series of similar samples are in progress. Pre-experiments indicate that SKP can detect the migration front, that visible in SAM images.

For the linear rather than square-root time dependence of the propagation of the migration front different explanations are conceivable. As mentioned in the introduction, the linear growth can be related to a type II diffusion [21, 22, 40], where the diffusion is self-accelerated by structural changes due to the diffusing species. This may be caused by a decrease of the glass transition temperature caused by swelling and hydroplastification. Another mechanism of self-acceleration is a stressdepending diffusion coefficient caused by swelling. As discussed above, the 'historydependent' diffusion shows a close analogy to the mechanisms determining the linear growth of a crazing front during mechanical deformation [23, 24]. Similar processes are proposed to describe the linear growth of cracks [41].

Also, electrochemical mechanisms could be responsible for an accelerated diffusion. The facilitated transport of ions in an electrical field gradient $[2,3]$ might be such a process. It has been shown by Reddy and Sykes $[25,26]$ that after cathodic reduction of rust at a defect the site "switches", becoming anodic and the cathodic region advances outward with new anodic sites developing in front of it. The authors state that around the defect easier pathways for currents of ions can develop due to the adhesion loss, thereby making high local currents underneath the coating possible $[25,26]$. The potential gradients that result from the cyclic cathode/anode switches combined with the easier pathways could be responsible for the fast migration that was detected in our sample.

The finding of a constant migration velocity seems to be in contradiction to other experiments, that use destructive measuring methods, which have found that diffusion of ions (following a square-root dependence) is the rate-determining process for disbonding $[5,7,8]$. However, it has to be mentioned, that in most of those experiments the extent of the "cathodic delamination" was determined by visual inspection [3,8], whereas the ultrasonic microscope detects changes in the acoustic properties caused by the penetrating fluids. Chemical processes resulting in delamination and corrosive products (obvious in the visual inspection) are not necessary to get acoustic contrast in SAM. Therefore, visual inspection and SAM may detect different processes, which obey different time characteristics. Furthermore, the different polymeric binders can lead to different diffusion mechanisms, as the high glass transition temperature and the strong adhesive strength of epoxy coatings probably impedes self-accelerated transport mechanisms, for example. 


\subsection{Blister growth kinetics}

As shown above, blister nucleation and growth can take place in samples with clear coat, in areas, where the migration front, and therefore water, has already been present for some time. Fig. $6 \mathrm{~b}-\mathrm{f}$ shows the formation and growth of blisters in the migration zones for sample BC/CC/D2. From the images it is apparent that the first blisters appear along the edges and close to the initial defect, with new blisters forming close to the migration front. So the process of blister formation is restricted to areas of prior exposure to water, dissolved oxygen and the reaction products resulting from the reduction of the oxygen.

Despite the error resulting from the difference between ultrasonically visible and actual size due to sound scattering and diffraction, the mean radius of selected blisters was measured for different immersion times. The results are shown in Fig. 9. For reasons of clarity the error bars were omitted in the graph. The error of the measurements was between $7-15 \%$.

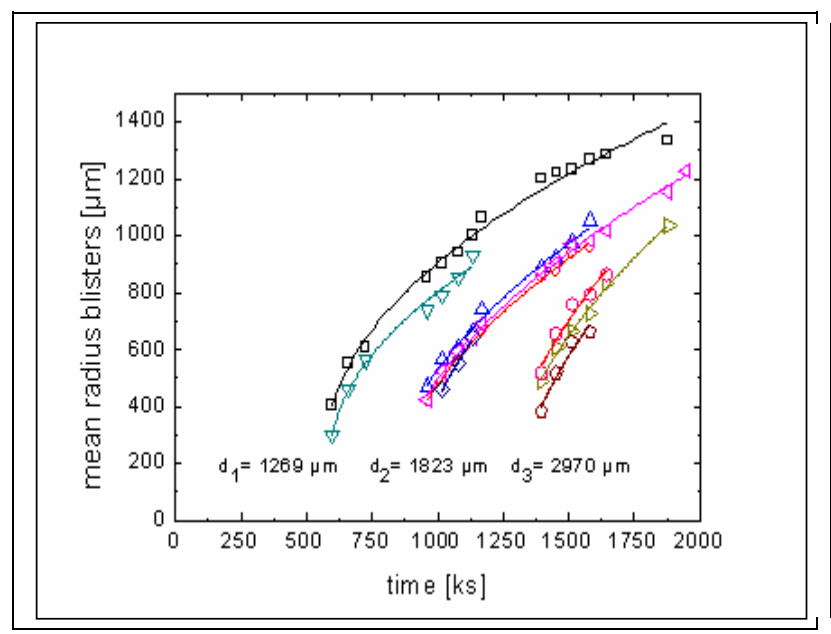

Fig.9: $\quad$ Time dependence of the mean radius of blisters. The lines represent fits yielding exponents of $0.5 \pm 0.02$. The mean distances of the blisters from the edges for the three blister groups $d_{1}-d_{3}$ are indicated below each group.

The data in Fig. 9 were fitted using a power law:

$$
x=a \cdot\left(t-t_{0, b}\right)^{p}
$$

yielding an exponent of $p=0.5 \pm 0.02$ for all curves, which indicates a diffusion controlled transport mechanism. $t_{0, b}$ is the induction time, $x$ the mean blister radius and $a$ is a fitting parameter. It should be noted that the blister growth process being discussed was detected on the identical sample for which the constant velocity for the propagation of the migration front $v_{\text {migr }}$ was found. Therefore it can be concluded that the two observed effects result from different mechanisms or a different combination of mechanisms and are not caused by different experimental conditions. As can be seen in Fig. 9 the blistering does not immediately start with the immersion of the sample. This is represented by an induction time in equation 4 . Induction times 
before cathodic blistering have been found before [7]. The data in Fig. 9 also shows that the blisters can be grouped according to their approximate induction time. These are about the same for all blisters in the same group. In order to relate the induction times for the different groups to the migration front, the distance of the blisters to the edge was measured. The values were in a close range for each group. A linear fit of the dependence of the mean induction time of the blister groups on their mean distance to the edge (Fig. 10a) yields a slope value of $400 \mathrm{~s} / \mu \mathrm{m}$, which leads to a reciprocal value of $v_{\text {blisterfront }}=2.5 \mathrm{~nm} / \mathrm{s}$. This corresponds within the error limits to the velocities calculated for the migration fronts $v_{\text {migr }}$ of $1.7-3.6 \mathrm{~nm} / \mathrm{s}$ measured for the identical sample. This supports the assumption that water and possibly ions have to be transported to a given position first, before blistering starts there. Hence, the weak wet adhesion and most probably the cathodic processes starting in those regions are a prerequisite for the initiation or "nucleation" of cathodic blistering.

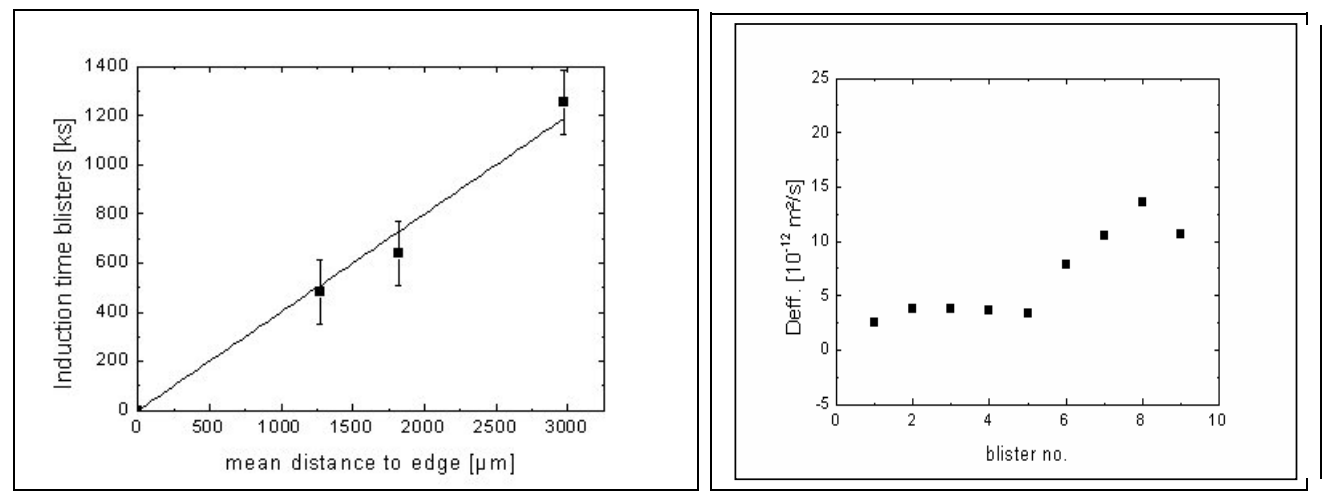

Fig. $10 a-b:$ a) Dependence of the induction times on the mean distance to the edge and b) effective diffusion coefficients resulting from the non-linear fits in Fig. 7.

The effective diffusion coefficients calculated for the selected blisters are shown in Fig. $10 \mathrm{~b}$, they range from $2.83-13.62 \times 10^{-12} \mathrm{~m}^{2} / \mathrm{s}$ with errors of $10-20 \%$. The values correspond in order of magnitude to diffusion coefficients of water in epoxypolyamine and rubber modified systems described elsewhere [2, 4, 11, 42].

\section{Conclusion}

In this article it has been shown that ultrasonic microscopy is a method capable of detecting fluid penetration and migration leading to weak wet adhesion and coating failure due to cathodic delamination prior to any visual delamination that occurs after exposure to a corrosive environment. Different damage mechanisms - penetration and migration of water resulting in weak wet adhesion, leading to disbonding, delamination and blister formation - could be identified.

The experiments conducted revealed a dependence of the occurring mechanisms on the layer structure and the depth of an initial defect, whereas no dependence on the form of the initial defect could be detected. As expected the presence of a clear coat was found to be important for the protecting properties of a coating layer structure. For samples without clear coat and primer layer, statistically distributed nucleation of micro-blisters on the metal substrate was observed. In samples with clear coat, migration fronts were detected which started from initial defects and the unprotected edges of the sample. For the systems with clear coat, initial defect depth is important, 
since defects exposing the substrate lead to migration fronts and subsequent disbonding. The measurements also confirm that the migration and disbonding process takes place in the substrate/coating interface region.

For the samples with a clear coat the ultrasonic images show two distinct processes on the same sample: (i) migration and disbonding and (ii) blister nucleation and growth. The migration process was found to be linear with time with the fronts propagating with velocities of $1.7-3.6 \mathrm{~nm} / \mathrm{s}$. The migration velocities for $x$ - and $y$ direction differed, which can be explained by the morphology of the substrate (being most probably due to the rolling direction of the steel).

The kinetics of the subsequent growth of blisters has been identified to be a diffusioncontrolled process with power law exponents of 0.5 . The blister nucleation induction times were matched with the migration front reaching the nucleation sites. This supports the theory that the presence of water and the resulting corrosive cathodic processes are prerequisites for the nucleation of blisters. Effective diffusion coefficients for blister growth were estimated yielding values of $2.83-13.62 \times 10^{-12} \mathrm{~m}^{2} / \mathrm{s}$.

Although the method has its limitations in that it does not allow the investigation of separate layers or interfaces of polymers with similar acoustic impedance, the results show that SAM is nonetheless suited for the investigation of transport mechanisms and defect propagation in coating systems. For a better understanding of the underlying processes and their kinetics, further experiments with model systems as well as corroboration of the results by other measuring methods are needed. Also, further improvement of the SAM data analysis - as for example the evaluation of the surface topography that was carried out - would be helpful.

\section{Acknowledgment}

Financial support from the Federal Ministry of Economics and Technology (BMWi) through the Federation of Industrial Cooperative Research Associations "Otto von Guericke" (AiF) (IGF-No. 15311 N) is gratefully acknowledged. 


\section{References:}

[1] Sorensen, P.A.; Kiil, S.; Dam-Johansen, K.; Weinell, C.E: Journal of Coating Technology and Research Vol. 6, No. 2 (2009). 135.

[2] Sorensen, P.A.; Dam-Johansen, K.; Weinell, C.E.; Kiil, S.: Progress in Organic Coatings Vol. 68 (2010). 70.

[3] Sorensen, P.A., Dam-Johansen, K.; Weinell, C.E.; Kiil, S.: Progress in Organic Coatings Vol. 68 (2010). 283.

[4] Liu, M.; Wu, P.; Ding, Y.; Chen, G.; Li, S.: Macromolecules Vol. 35 (2002) 5500.

[5] Parks, J.; Leidheiser, H.: Industrial and Engineering Chemistry Product Research and Development Vol. 25 (1986) 1.

[6] Bajat, J.B.; Milosev, I.; Jovanovic, Z.; Miskovic-Stankovic, V.B.: Applied Surface Science Vol. 256 (2010) 3508.

[7] Crossen, J.D.; Sykes, J.M.; Zhai, T.; Briggs, G.A.D.: Faraday discussions Vol. 107 (1997) 417.

[8] Sorensen, P.A.; Kiil, S.; Dam-Johansen, K.; Weinell, C.E.: Progress in Organic Coatings Vol. 64 (2009) 142.

[9] Alig, I.; Tadjbach, S.; Krüger, P.; Oehler, H.; Lellinger, D.: Progress in Organic Coatings Vol. 64 (2009) 112.

[10] Horner, M.R.; Boerio, F.J.: The Journal of Adhesion Vol. 32 (1990) 141.

[11] Armstrong, R.D.; Johnson, B.W.; Wright, J.D.: Electrochimica Acta Vol. 36 (1991) 1915.

[12] Weinell, C. E.; Rasmussen, S. N.: Non-destructive determination of rust creep. NACE International, Corrosion 2008 - Conference \& Expo. Paper No. 08004 (2008).

[13] Leidheiser, H.; Kendig, M.W.: Corrosion Vol. 32 (1976) 69.

[14] Funke, W.: Industrial and Engineering Chemistry Product Research and Development, Vol. 24 (1985) 343.

[15] Funke, W.: Progress in Organic Coatings, Vol. 9 (1981) 29.

[16] Momber, A.: Blast Cleaning Technology. Springer Verlag Heidelberg (2008) 453.

[17] Leng, A., Streckel, H, Stratman M.: Corrosion Science, Vol. 41 (1999) 599. 
[18] Crank, J., Park, G.S.: Transaction of the Faraday Society, Vol. 47 (1951) 1072.

[19] Crank, J.: Journal of Polymer Science, Vol. 11 (1954) 151.

[20] Gostoli, G.; Sarti, G.C.: Polymer Engineering and Science, Vol. 22, No. 16 (1982) 1018.

[21] Peterlin, A.: Die makromolekulare Chemie, Vol. 124, No. 3009 (1969). 136.

[22] A. Peterlin: Journal of Polymer Science, Vol. 17 (1969).

[23] Sarti, G. C.: Polymer, Vol. 20 (1979) 827.

[24] J. A. Hsiao, C. C. Sauer: Transactions of the ASME, Vol. 75 (1953) 895.

[25] Reddy, B.; Doherty, M.J.; Sykes, J.M.: Electrochimica. Acta Vol. 49 (2004) 2965.

[26] Reddy, B.; Sykes, J.M.: Progress in Organic Coatings Vol. 52 (2005) 280.

[27] Dornbusch, M.: Progress in Organic Coatings Vol. 61 (2008) 240.

[28] Deflorian, F.; Fedrizzi, L.: Journal of Adhesion Science and Technology Vol.13, No.5 (1999) 629.

[29] Brand, S.; Raum, K.; Czurratis, P.: Ultrasonics Symposium (2008) 615.

[30] Briggs, G.A.D.: Acoustic Microscopy, Clarendon Press, Oxford (1992).

[31] Koehler, E.L.: Corrosion Vol. 33 (1977) 209.

[32] Briggs, G.A.D.: An Introduction to Scanning Acoustic Microscopy, Oxford University Press/Royal Microscopy Society, Oxford (1985).

[33] Anderton, W.: Official Digest - Federation of Societies of Paint Technology Vol. 36 (1964) 1210.

[34] Hammond, J.S.; Holubka, J.W.; Dickie, R.A.: Journal of Coatings Technology Vol. 51 (1979) 45.

[35] Addison Jr., R.C.; Kendig, M.W.; Jeanjaquet, S.J.: Proceedings IEEE, Ultrasonics Symposium Vol. 2 (1988) 739.

[36] Atkins, P.W.: Physical Chemistry, W.H. Freeman \& Co., New York (1997).

[37] Hilliard, J, Cahn, J.: Acta Metallurgica Vol. 6 (1958). 772. 
[38] Cahn, J.: Acta Metallurgica Vol. 8 (1960) 554.

[39] Lange, J., Manson, J-A.E.; Hult, A.: Journal of Coatings Technology Vol. 66, No. 838 (1994) 19.

[40] Peterlin, A.: Journal of Polymer Science: Polymer Physics Edition Vol. 17 (1979) 1741.

[41] Ravi-Chandar, K.; Knauss, W.G.: International Journal of Fracture Vol. 26 (1984). 141.

[42] Cabanelas, J.C.; Prolongo, S.G.; Serrano, B.; Bravo, J.; Baselga, J.: Journal of Materials Processing Technology Vol. 143-144 (2003) 311. 